\title{
An Architectural Mechanism for Resilient IoT Services
}

\author{
Hokeun Kim \\ University of California, Berkeley \\ hokeunkim@eecs.berkeley.edu \\ David Broman \\ KTH Royal Institute of Technology \\ dbro@kth.se
}

\author{
Eunsuk Kang \\ University of California, Berkeley \\ eunsuk@berkeley.edu \\ Edward A. Lee \\ University of California, Berkeley \\ eal@eecs.berkeley.edu
}

\begin{abstract}
Availability of authentication and authorization services is critical for the safety of the Internet of Things (IoT). By leveraging an emerging network architecture based on edge computers, IoT's availability can be protected even under situations such as network failures or denial-of-service (DoS) attacks. However, little has been explored for the issue of sustaining availability even when edge computers fail. In this paper, we propose an architectural mechanism for enhancing the availability of the authorization infrastructure for the IoT. The proposed approach leverages a technique called secure migration, which allows IoT devices to migrate to other local authorization entities served in trusted edge computers when their authorization entity becomes unavailable. Specifically, we point out necessary considerations for planning secure migration and present automated migration policy construction and protocols for preparing and executing the migration. The effectiveness of our approach is illustrated using a concrete application of smart buildings and network simulation, where our proposed solution achieves significantly higher availability in case of failures in some of the authorization entities.
\end{abstract}

\section{CCS CONCEPTS}

- Computer systems organization $\rightarrow$ Availability; Fault-tolerant network topologies; $\bullet$ Security and privacy $\rightarrow$ Authentication; Authorization; Denial-of-service attacks;

\section{KEYWORDS}

Internet of Things, Network security, Availability, Denial-of-service attacks, Authorization, Authentication

\section{ACM Reference Format:}

Hokeun Kim, Eunsuk Kang, David Broman, and Edward A. Lee. 2017. An Architectural Mechanism for Resilient IoT Services. In Proceedings of Proceedings of the First ACM Workshop on the Internet of Safe Things (SafeThings'17). ACM, New York, NY, USA, 6 pages. https://doi.org/10.1145/3137003.3137010

Permission to make digital or hard copies of all or part of this work for personal or classroom use is granted without fee provided that copies are not made or distributed for profit or commercial advantage and that copies bear this notice and the full citation on the first page. Copyrights for components of this work owned by others than ACM must be honored. Abstracting with credit is permitted. To copy otherwise, or republish, to post on servers or to redistribute to lists, requires prior specific permission and/or a fee. Request permissions from permissions@acm.org.

SafeThings'17, November 5, 2017, Delft, The Netherlands

(C) 2017 Association for Computing Machinery.

ACM ISBN 978-1-4503-5545-2/17/11 ..\$15.00

https://doi.org/10.1145/3137003.3137010

\section{INTRODUCTION}

Authentication and authorization play key roles in ensuring safe and secure operations of the Internet-of-Things (IoT) devices in an open, potentially malicious environment. In the context of the IoT, most of existing approaches to providing authorization and authentication rely on remote and centralized cloud servers to provide these services. This means, however, that any device relying on these critical services may be adversely affected by a failure or an attack on one or more of the servers. An example illustrating this risk is the recent Google OnHub incident [14], where a failure in the company's authentication servers caused IoT gateway devices (called $\mathrm{OnHub}$ ) to become unavailable, in turn leading to failures in all IoT devices connected to these gateway devices.

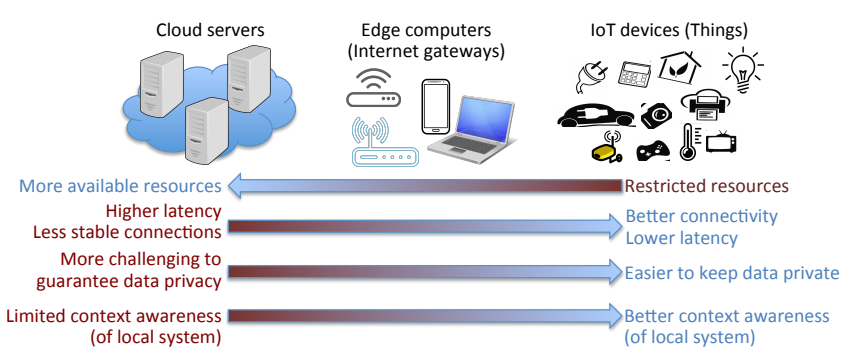

Figure 1: Characteristics of cloud, edge, and things

An alternative system architecture, in part designed to reduce this dependency on remote, centralized servers, is called edge computing [21] or fog computing [11]. In this approach, individual computing devices called edge computers serve as an Internet gateway to local, neighboring devices; these edge computers may be deployed as a wide range of devices, including smart home routers, laptops, or smart phones. Figure 1 illustrates different characteristics of the cloud servers, edge computers, and IoT devices. As pointed out by Lopez et al. [6], benefits of adopting edge computing include better privacy, lower latency for real-time applications, less dependency on cloud servers and its connections, and better context awareness and manageability of the local systems.

Previously, we proposed an authorization infrastructure of the IoT called Secure Swarm Toolkit (SST) [8]. SST leverages a network architecture with special edge computers called Auths, which provide authorization services to local IoT devices. In this prior work, we showed how SST can provide strong confidentiality and integrity guarantees for communication among IoT devices.

In this paper, building on our prior work, we propose a novel approach for providing resilience against availability attacks or 
failures on an IoT network-e.g., a denial-of-service (DoS) attack designed to render critical services such as authorization unavailable to IoT devices. In particular, our approach leverages a technique called secure migration, which allows IoT devices to continue to receive critical services even when some of the Auth devices become unavailable due to an attack or other failures.

The contributions of this paper are as follows:

- An innovative architectural mechanism for building IoT services that are resilient against availability attacks and other failures, including a secure migration technique which allows IoT entities to migrate to trusted authorization entities to continue IoT services when some of the authorization entities are unavailable.

- A novel approach for finding effective migration policy by leveraging a Integer Linear Programming (ILP) solver and considering access requirements and constraints of IoT networks (Section 3).

- Experiments based on a concrete scenario in smart buildings, demonstrating the effectiveness of our approach against availability attacks (Section 4).

\section{PROBLEM AND CONTEXT}

IoT devices are heterogeneous, in that they vary over the level of safety and security requirements as well as available computational resources and energy capacity. For example, a smart door lock that authenticates users with their cellphones may rely on secure communication with strong encryption, whereas temperature sensors in the same building may not require the same level of security.

Important parts of an IoT infrastructure are special devices called gateways. A gateway facilitates communication between IoT devices by providing a variety of services such as authentication, authorization, device discovery, and data aggregation. Depending on the design of the infrastructure, a gateway may be deployed as a cloud-based server or as a local network entity (e.g., a router) to which IoT devices can connect to.

\subsection{Threat Model}

We assume the existence of an active network attacker with an ability to discover and send packets to gateway devices on the Internet. In particular, the attacker seeks to disrupt the availability of IoT devices connected to a gateway by carrying out various denial-of-service (DoS) attacks on it, such as flooding or distributed denial-of-service (DDoS) attacks. In our model, we primarily focus on availability attacks and failures, instead of confidentiality or integrity attacks (addressed by our previous work, SST [8]).

In addition, we exclude insider attacks; i.e., malicious acts by an individual who has physical or network access to IoT and gateway devices on the local area network. For instance, we assume that a local gateway placed inside a building is protected from physical tamperings, and that local network users are trustworthy. This allows us to assume that local gateways can be leveraged to perform critical steps in mitigating against an availability attack.

\subsection{Proposed Architectural Mechanism}

The key distinguishing feature of SST is a locally centralized, globally distributed architecture [9]: It consists of a set of local authorization entities called Auths [10], each of which is deployed on edge computers. An Auth enforces access policies and provides security

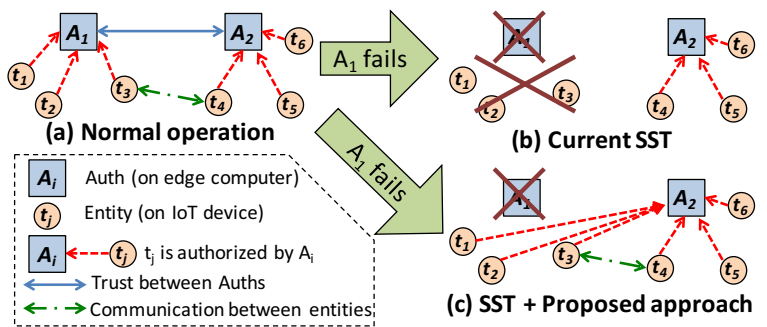

Figure 2: (a) SST in normal operation (b) Current SST in case of Auth failure (c) Proposed approach for enhancing availability of SST in case of Auth failure

services to local IoT devices (entities). Figure 2 (a) shows an example of a small IoT network in normal operation using SST, with two Auths $\left(A_{1}\right.$ and $\left.A_{2}\right)$ and 6 entities $\left(t_{1}\right.$ through $\left.t_{6}\right)$. If $A_{1}$ in the current SST design fails due to a DoS attack, authorization services for registered entities will become unavailable as shown in Figure 2 (b). This will also affect availability of other entities. For example, communication between $t_{4}$ and $t_{3}$ will be disrupted.

However, this does not fully utilize the globally distributed architecture of SST. Our goal is to provide enhanced availability of IoT authentication and authorization services through a mechanism that leverages SST's globally distributed architecture. The architectural mechanism proposed in this paper includes backing up information for authorization services to other trusted Auths and securely migrating IoT entities to continue the IoT services even in case of Auth failures as shown in Figure 2 (c). The proposed mechanism is detailed in Section 3.

\section{APPROACH}

We propose an approach called a secure migration technique for restoring the availability of IoT devices during failures in some Auths by exploiting the architectural characteristics of SST. In particular, we accomplish this goal by having Auths take over other Auths' authorization tasks when the latter group becomes unavailable due to an attack. Before any failure occurs in Auths, each Auth backs up information and necessary credentials to trusted Auths. When an Auth failure occurs, its registered entities detect the failure and migrate to other trusted Auths as depicted in Figure 2 (c) so that they can continue to use authorization services.

\subsection{Considerations for Migration Policies}

Although the high-level idea behind migration may appear straightforward, determining migration policies is a non-trivial problem due to a number of factors that need to be considered:

Auth trust relationships: SST introduces an explicit notion of trust between a pair of Auths, and enables an entity from Auth $A$ to communicate to an entity in another Auth $A^{\prime}$ if and only if $A$ and $A^{\prime}$ trust each other. This communication constraint imposed by trust must be maintained during migration while satisfying individual entities' requirements. Consider Figure 3 (a), where entities $t_{4}$ and $t_{5}$ are required to communicate with each other. Suppose that $A_{1}$ becomes unavailable. If we arbitrarily decide to migrate $t_{4}$ to $A_{2}$, then $t_{4}$ will no longer be allowed to communicate with $t_{5}$, since 


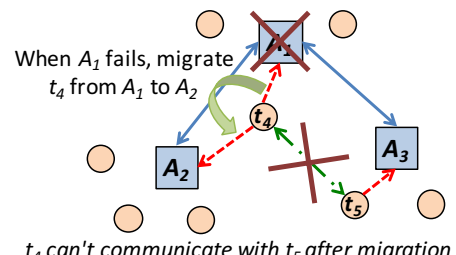

(a)

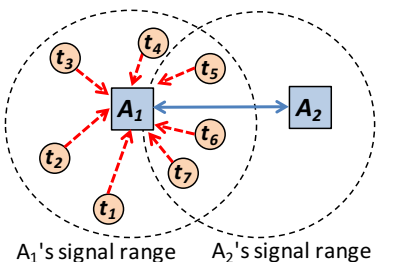

(b)

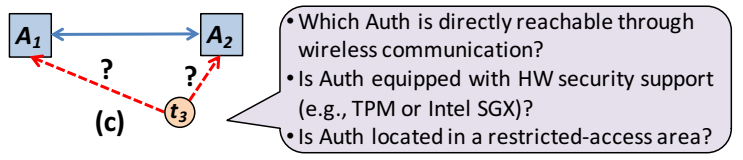

Figure 3: Considerations for migration policies (a) Trust among Auths (b) Workload for Authorization (c) Auth's characteristics (Figure legends are the same as Figure 2)

there exists no trust relationship between $A_{1}$ and $A_{3}$. A proper migration policy may instead involve migrating $t_{4}$ to $A_{3}$. Note that the trust relationship among Auths is not necessarily transitive.

Managing Auth's workload for authorization: Let us consider a different scenario where entities who have lost connection with their original Auths are placed within the signal ranges of Auths $A_{1}$ and $A_{2}$ as depicted in Figure 3 (b). In this scenario, $t_{5}, t_{6}$ and $t_{7}$ are within signal ranges of both Auths, thus they can migrate to either of Auths. An arbitrary migration decision may lead to a situation described in Figure 3 (b) where all 7 entities will have migrated to only $A_{1}$. However, this type of decision may adversely affect the performance of the overall network, depending on the operational conditions of Auths. Each Auth may have an inherent limit in its resource capacity to perform authorization tasks for entities, for example, $A_{1}$ may be required to run other computationallyintensive tasks, or it may be running on a battery-powered edge computer with a limited energy budget. Therefore, assigning entities within $A_{2}$ 's signal range $\left(t_{5}, t_{6}\right.$ and $\left.t_{7}\right)$ to $A_{2}$ may lead to a greater performance for the overall network.

Characteristics of Auth: Another important factor to be considered in a migration policy is various aspects of Auth from the viewpoint of each entity, as shown in Figure 3 (c). One example is reachability, which includes whether an Auth supports underlying network protocols that an entity is using and whether Auth is within the wireless signal range. Another example of Auth characteristics is the levels of security guaranteed by an Auth, compared with those required by an entity. These include whether the Auth is equipped with hardware security support such as a TPM (Trusted Platform Module) or Intel's SGX (Software Guard Extensions) and whether the Auth is located in a restricted-access area for extra physical security.

\subsection{Policy Construction}

When an Auth becomes unavailable due to an attack, we say that entities belonging to that Auth are dangling. A migration policy is an assignment of each dangling entity to one of the remaining Auths in the network. As discussed in Section 3.1, a valid policy must ensure that the new network resulting from migration satisfies a number of hard constraints (e.g., trust relationships).
We formulate the problem of constructing a valid migration policy as a problem in Integer Linear Programming (ILP). An ILP problem has two parts: a set of linear terms (each term being a product of an integer variable by a coefficient; e.g., $c_{i} x_{i}$ for some variable $x_{i}$ ) whose sum is to be maximized, and a set of linear constraints over those variables. In our formulation, variables are Boolean values indicating whether an entity is connected to an Auth (thus, there are in total $E \times A$ number of variables, where $E$ and $A$ are the total numbers of entities and Auths in the network, respectively).

Due to limited space, we omit a detailed formalization of the ILP problem, and instead briefly describe the set of constraints that must be satisfied by a solution:

- Each entity can have security requirements that must be provided by an Auth. These requirements include hardware support (e.g., TPM/SGX), restricted physical location, cryptography specification (e.g., ephemeral keying for perfect forward secrecy).

- Each Auth has a threshold for authorization tasks in terms of upper bounds for authorization requests per minute or upper bounds for session keys cached in Auth. In other words, there is a threshold of registered entities for each Auth. When there are more registered entities than this threshold the Auth will experience degradation in authorization.

- Entity-to-entity communication requirements. Some entities need to be authorized to communicate with certain entities even after migration. This requires trust relationship between Auths with which those entities are registered.

- There exist multiple criticality levels for the communication requirements among entities, yielding a mixed-criticality system [4]. This means we should prioritize communication requirements of high-criticality entities.

Another aspect of policy construction is that of optimization; that is, generating a migration policy that results in a network with a minimal amount of overall communication costs between Auths and things. For instance, communication costs between a pair of nodes may depend on a number of factors, such as the physical distance between them and the energy required to perform cryptographic operations. Our formulation of policy construction as an ILP is intended to enable optimization of migration policies as well. This task, however, requires a detailed modeling of communication and energy costs, which is beyond the scope of this paper.

For our current implementation of SST, we use Gurobi [7] as the underlying ILP solver.

\subsection{Secure Migration Procedure}

In SST, each entity is authorized by Auth by receiving a session key, which is a temporary symmetric cryptographic key for accessing a certain service or communicating with another entity (or entities). Session keys need to be confidential; they must be only known to the entities participating in a certain access activity. Thus, these session keys must always be encrypted with another symmetric key called a distribution key shared between Auth and each entity.A distribution key can be updated using public-key cryptography. For this, Auth and the registered entity should have already exchanged public keys during the registration (initialization) process of SST. For secure migration, Auth must prepare the migration before it 


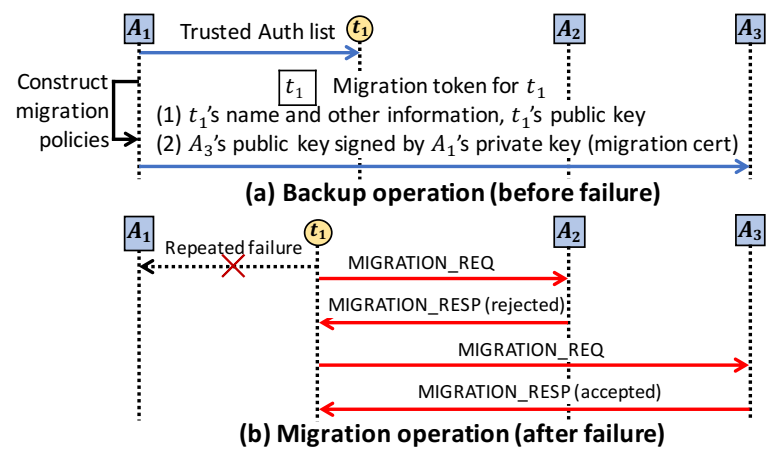

Figure 4: Secure migration procedure (a) Backup operation: $A_{1}$ updates its Thing with a trusted Auth list and sends a migration token after migration policy construction (b) Migration operation: $A_{1}$ fails and its entity $t_{1}$ tries to migrate to $A_{2}$ or $A_{3}$.

fails. When a failure occurs, Auth's registered entities should detect the failure and migrate to trusted Auths. The approach described here is implemented as part of SST and available in SST's opensource repository (https://github.com/iotauth).

3.3.1 Preparing Migration. Before a failure occurs, Auth should provide both its registered entities and the trusted Auths with information for migration. We call this operation a backup operation, described in Figure 4 (a). For its registered entity $t_{1}$, Auth $A_{1}$ gives out a list of its trusted Auths $\left(A_{2}\right.$ and $\left.A_{3}\right)$ and their host names (IP addresses) and port numbers. This is first given during the entity registration and then updated when the information changes. Then, $A_{1}$ constructs the migration policies for its registered entities, either by itself or by receiving migration policies from other trusted Auths, using the method shown in Section 3.2.

With the constructed migration policies, $A_{1}$ prepares information and credentials, migration token for each of its registered entities. A migration token must be able to establish the trust relationship between entities and the new Auth, $A_{3}$, to which $t_{1}$ will migrate in case of $A_{1}$ 's failure. $A_{1}$ includes the name (a unique identifier in string) and the public key of $t_{1}$ so that $A_{3}$ can trust $t_{1}$ when it migrates to $A_{3}$, as shown in Figure 4 (a)-(1). Auth also issues a migration certificate (cert) shown in Figure 4 (a)-(2) for $A_{3}$. This includes $A_{3}$ 's public key signed by $A_{1}$ 's private key and this will be verified by $t_{1}$ which already has $A_{1}$ 's public key.

3.3.2 Detection and Migration. When Auth $A_{1}$ fails, its registered entity $t_{1}$ will detect that $A_{1}$ is not reachable due to a failure or an attack and will try to migrate to $A_{1}$ 's trusted Auths. When $A_{1}$ does not respond for more than a threshold times, $t_{1}$ starts a migration operation shown in Figure 4 (b). Since $A_{1}$ had provided $t_{1}$ with a list of $A_{1}$ 's trusted Auths which were $A_{2}$ and $A_{3}, t_{1}$ tries these Auths in the order specified in the list. Thus, $t_{1}$ sends MIGRATION_REQ to $A_{2}$, an Auth trusted by $A_{1}$, but not the one that $t_{1}$ is supposed to migrate to. Since $A_{2}$ does not have the required credentials for $t_{1}, A_{2}$ sends a response, MIGRATION_RESP indicating that the request is rejected and $t_{1}$ should try another trusted Auth in its list. After receiving MIGRATION_RESP (rejected) from $A_{2}, t_{1}$ tries the next Auth in its list, $A_{3}$. When $A_{3}$ receives MIGRATION_REQ

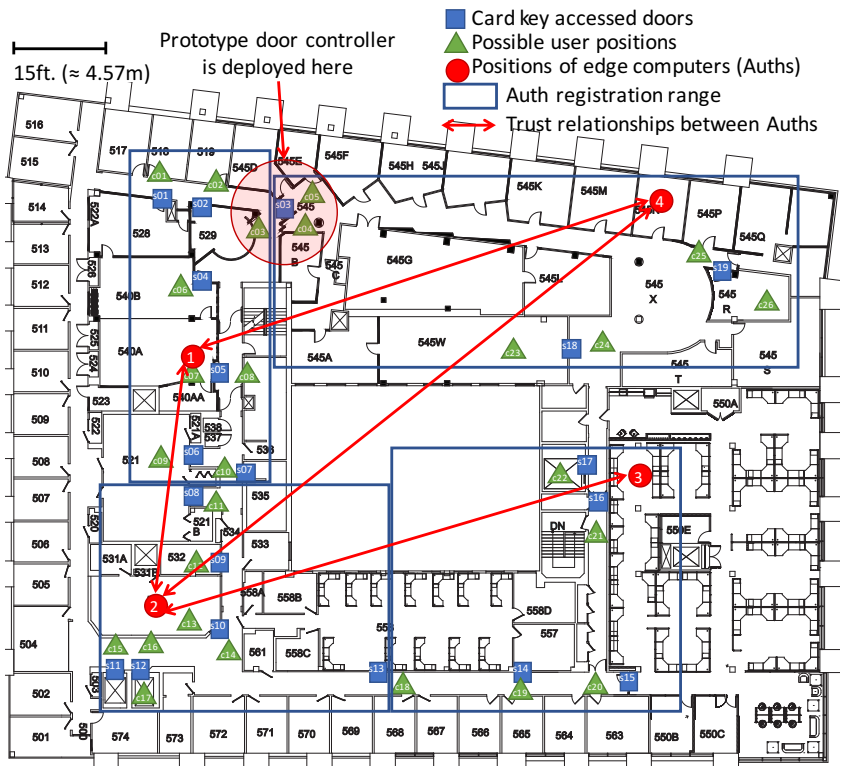

Figure 5: Experimental virtual environment with Auths, door controllers, and door opening mobile applications on the floor map of the 5th floor, Cory Hall at UC Berkeley

from $t_{1}$, it notices that $t_{1}$ can migrate to it and responds with a MIGRATION_RESP message indicating that the request has been accepted.

A MIGRATION_REQ message includes the entity's name and a verification token. This verification token includes a digital signature of the entity if it uses public-key cryptography for authorization. The Auth that the entity is supposed to migrate to will be able to verify the MIGRATION_REQ with the entity's public key. This is because the Auth should have been backed up with the entity's public key from the Auth that the entity was previously registered with. The accepted MIGRATION_RESP contains the certificate of the $A_{3}$ signed by $A_{1}$. The whole MIGRATION_RESP should be authenticated by the Auth's private key, so that the entity can verify the MIGRATION_RESP message upon receiving it. When the migration request and response are successful, both the Auth and entity update the counterpart's credentials for further authorization.

\section{EXPERIMENTS AND RESULTS}

In this section, we carry out experiments to demonstrate the effectiveness of our migration approach for maintaining availability. As an experimental scenario, we take a door controller and door opening application in a smart building. This is inspired by a prototype door controller deployed on the 5th floor of Cory Hall at UC Berkeley. We assume a virtual environment where the door controllers are deployed on currently card-key accessed doors, door opening mobile phone apps run on user smart phones, and Auths are deployed on some of the existing WiFi access points. Figure 5 illustrates this virtual environment. We also assume Auths trust each other only if their research centers have trust relationships, and the Things (door controllers and user smart phones) are registered with Auth as shown in Figure 5. We measure availability 


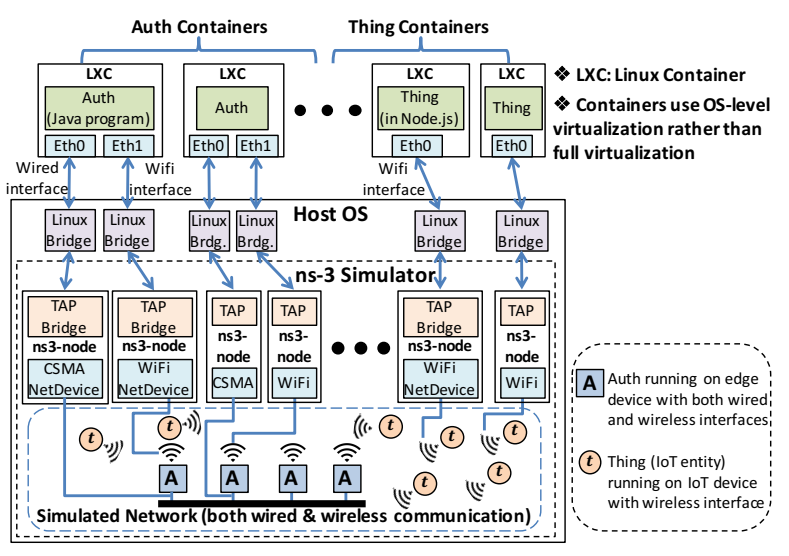

Figure 6: Experimental setup with the ns-3 simulator network simulator

as the ratio of responses from door controllers (the number of correct door operation) to the door opening requests for a given time window. In our experimental scenarios, different numbers of Auths can be unavailable due to failures or DoS attacks. In addition, we also compare against scenarios without secure migration and also a scenario where all Auths are unavailable, which is equivalent to the case where an authorization entity is deployed on a remote cloud and the cloud is not reachable.

\subsection{Experimental Setup}

Figure 6 describes the experimental setup with the ns-3 network simulator [19]. For realistic experiments, we use the actual implementation of Auth available on the GitHub repository and IoT entities (door controllers and opening apps) written using SST's APIs, secure communication accessors. Each of Auths and IoT entities runs within an individual Linux Container (LXC) [2] which provides OS-level virtualization (paravirtualization) with a separate virtual network space. For simulating the network infrastructure, we use ns-3. The LXCs' virtual Ethernet interfaces are connected to the host OS's Linux bridges, then to the TAP bridges of ns-3 nodes in the ns-3 simulator. The other side of ns-3 nodes are either CSMA or WiFi NetDevice and are connected to the network simulated in ns-3. LXCs on which Auths are running have both the wired and WiFi connections and LXCs for IoT entities have WiFi connections. For connections between Auths' wired network interfaces, we use a CSMA channel with data rate $100 \mathrm{Mbps}$. For connections between the wireless network interfaces of Auths and Things we use an ad-hoc IEEE 802.11a channel with data rate of 54Mbps. For the channel signal strength model, we use a Log Distance Propagation Loss Model in ns-3 to represent the channels between Auths and things. As a simulation platform, we use Ubuntu Linux 16.04.2 LTS on Amazon's AWS EC2 with 4 CPUs, 16GB RAM, and 256GB SSD.

\subsection{Simulation Results}

We ran simulations with 4 Auths, 19 door controllers, and 26 user devices for 15 minutes for each experiment in real time, 2.5 minutes before Auth failures and 12.5 minutes after failures. Each user device sends a door opening request to the closest door controller every minute, simulating the user behaviors. Figure 7 illustrates the experimental results. We performed experiments with up to three Auths failing during the experiments. The failure occurs in order of Auth 1, Auth 3, and Auth 4 where the Auth numbers are as shown in Figure 5. When all four Auths failed, the availability became $0 \%$ in our experiments as we expected, although we did not include this in Figure 7. It will be the same when the authorization services based on the cloud lose connections with the cloud. We also compared three different migration policies, (1) without any secure migration (original SST, denoted as "No Migration"), (2) with a naïve migration policy where the user devices attempt to migrate to the nearest available Auth and so on (denoted as "Naïve Migration"), and (3) with a migration policy constructed using the proposed approach in Section 3.2 (denoted as "ILP-Based Migration" in the results).

As shown in Figure 7 (a), when one Auth fails the availability drops down to $69 \%$ without any migration, while the naïve migration and ILP-based migration policies recover the availability up to $92 \%$ and $100 \%$, respectively. The naïve migration policy could not recover $100 \%$ availability due to the fact that it did not appropriately consider communication requirements among IoT entities together with trust relationships between Auths. In fact, with the naïve migration policy, some clients ended up migrating to Auths that do not have trust relationships with the Auths for the servers with which the clients were supposed to communicate.

In Figure 7 (b), we can see that the availability drops down to $50 \%$ without any migration and fluctuates around 58\% with the naïve solution, while the proposed ILP-based solution recovers $81 \%$ of availability. Figure 7 (c) shows decreased availability around 27\% with no or the naïve migration policy and recovered availability of $54 \%$ with the proposed technique. The fluctuation with the naive migration policy was mainly due to the interference caused by infeasible migration requests and unbalanced workload among functioning Auths. The proposed ILP-based solution was not able to achieve $100 \%$ availability because some entities were out of the signal range of Auths. However, the proposed solution still achieved significantly higher availability compared to the other two cases.

\section{RELATED WORK}

We are aware of only a couple of other approaches that rely on an edge computing architecture for IoT security. In order to provide robust authorization of medical IoT devices without dependency on remote servers, SEA (Secure and Efficient Authentication and Authorization Architecture) [13] uses distributed smart e-health gateways as local authorization centers based on DTLS (Datagram TLS). TACIoT (Trust-aware Access Control for the IoT) [3] employs an architecture based on entities called IoT bubbles, which act as local units of authorization for the IoT, similar to Auths. As far as we know, while these approaches ensure confidentiality and integrity of communication among IoT devices, they do not provide resilience against availability attacks.

Beside the two mentioned in the previous paragraph, a number of other authorization and authentication services for IoT devices have been proposed [1, 5, 15, 22, 24]. However, all of these approaches rely on remote, cloud servers to provide the critical security services. We believe that the local, distributed nature of Auths enables a more lightweight, flexible defense against availability attacks: Our 


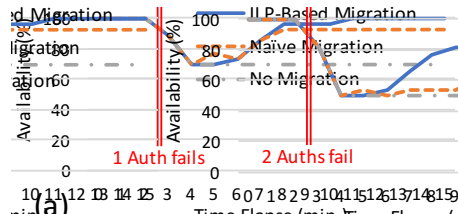

(a) $\begin{array}{lllllllllll}0 & 1 & 2 & 3 & 4 & 5 & 6 & 7 & 8 & 9 & 10 \\ & & & & & & \end{array}$

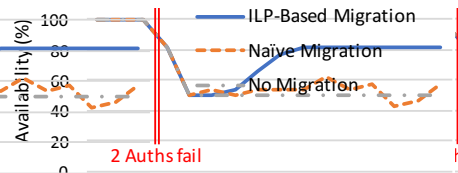

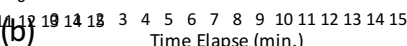
Time Elapse (min.)

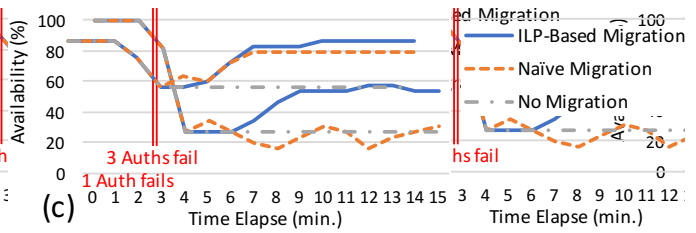

(c) $\begin{array}{lllllllllll}0 & 1 & 2 & 3 & 4 & 5 & 6 & 7 & 8 & 9 & 101112131415 \\ \text { Time Elapse (min.) }\end{array}$

Figure 7: Availability results with different numbers of failing Auths (a) 1 Auth fails (b) 2 Auths fail (c) 3 Auths fail, for three different migration policies using SST simulated on the ns-3 simulator and Linux containers

approach allows an Auth to continue providing critical services to its local network without requiring a connection to the Internet.

There exists a large body of work that address DoS attacks in the network security literature (a comprehensive survey can be found in [26]). These works can be categorized as (1) preventing an DoS attack before it takes place [12, 23, 27], (2) limiting the effect of an on-going attack by filtering malicious connections [16, 20], or (3) performing forensics after an attack to identify compromised IoT devices $[17,18,25]$. As far as we know, none of these approaches involve providing resilience against a DoS attack through migration of critical services from an attacked node to another.

\section{CONCLUSIONS}

Availability of IoT services can be critical for safety. By leveraging emerging network architecture based on edge computing and SST's distributed authorization infrastructure, the proposed approach achieves much higher availability even under failures of local authorization entities running on edge computers. Our secure migration approach will be appropriate especially for the Internet of Things under safety-critical environments including medical centers, manufacturing systems, and electric power grids, so that we can maintain as much availability as possible.

Moving forward, we plan to investigate the use of an ILP solver to construct a migration policy that is not only valid but also optimal with respect to the overall network costs. We also plan to carry out larger scale experiments to further evaluate the effectiveness and limitations of our migration mechanism. In addition, we plan to develop a mathematical model of an attacker, and explore possible relationships between the knowledge and capability of the attacker and the guarantees provided by our mitigation mechanism.

\section{ACKNOWLEDGMENTS}

This work was supported by STARnet, a Semiconductor Research Corporation program, sponsored by MARCO and DARPA. This work is also financially supported by the Swedish Foundation for Strategic Research (project FFL15-0032).

\section{REFERENCES}

[1] [n. d.]. How the AWS IoT Platform Works - Amazon Web Services. http://aws. amazon.com/iot-platform/how-it-works/

[2] [n. d.]. Linux Containers - LXC - Introduction. https://inuxcontainers.org/lxc/

[3] Jorge Bernal Bernabe, Jose Luis Hernandez Ramos, and Antonio F. Skarmeta Gomez. 2016. TACIoT: multidimensional trust-aware access control system for the Internet of Things. Soft Computing 20, 5 (May 2016), 1763-1779.

[4] Alan Burns and Rob Davis. 2015. Mixed criticality systems: A review. Dept. of Computer Science, University of York, Tech. Rep, Sixth Edition (Jan. 2015).

[5] Simone Cirani, Marco Picone, Pietro Gonizzi, Luca Veltri, and Gianluigi Ferrari 2015. IoT-OAS: An OAuth-Based Authorization Service Architecture for Secure Services in IoT Scenarios. IEEE Sensors fournal 15, 2 (Feb. 2015), 1224-1234.
[6] Pedro Garcia Lopez et al. 2015. Edge-centric Computing: Vision and Challenges. SIGCOMM Comput. Commun. Rev. 45, 5 (Sept. 2015), 37-42.

[7] Inc. Gurobi Optimization. 2016. Gurobi Optimizer Reference Manual. (2016). http://www.gurobi.com

[8] Hokeun Kim, Eunsuk Kang, Edward A. Lee, and David Broman. 2017. A Toolkit for Construction of Authorization Service Infrastructure for the Internet of Things. In The 2nd ACM/IEEE International Conference on Internet-of-Things Design and Implementation. ACM/IEEE, Pittsburgh, PA, 147-158.

[9] Hokeun Kim and Edward A. Lee. 2017. Authentication and Authorization for the Internet of Things. IT Professional 19, 5 (September 2017). to appear.

[10] Hokeun Kim, Armin Wasicek, Benjamin Mehne, and Edward A. Lee. 2016. A Secure Network Architecture for the Internet of Things Based on Local Authorization Entities. In The 4th IEEE International Conference on Future Internet of Things and Cloud. Vienna, Austria, 114-122.

[11] Tom H. Luan, Longxiang Gao, Zhi Li, Yang Xiang, Guiyi Wei, and Limin Sun. 2015. Fog Computing: Focusing on Mobile Users at the Edge. arXiv:1502.01815 [cs] (Feb. 2015). http://arxiv.org/abs/1502.01815 arXiv: 1502.01815.

[12] Parikshit N. Mahalle, Bayu Anggorojati, Neeli R. Prasad, and Ramjee Prasad. 2013. Identity Authentication and Capability Based Access Control (IACAC) for the Internet of Things. F. of Cyber Security and Mobility 1, 4 (2013), 309-348.

[13] Sanaz R. Moosavi et al. 2015. SEA: A Secure and Efficient Authentication and Authorization Architecture for IoT-Based Healthcare Using Smart Gateways. Procedia Computer Science 52 (Jan. 2015), 452-459.

[14] Ian Morris. 2017. Google's Latest Failure Shows How Immature Its Hardware Is. Forbes (Feb. 2017). http://www.forbes.com/sites/ianmorris/2017/02/ 24/googles-latest-failure-shows-how-immature-its-hardware-is/

[15] Antonio L. Maia Neto et al. 2016. AoT: Authentication and Access Control for the Entire IoT Device Life-Cycle. In Proc. of the 14th ACM Conf. on Embedded Network Sensor Syst. CD-ROM (SenSys '16). ACM, New York, NY, USA, 1-15.

[16] Luís M. L. Oliveira, Joel J. P. C. Rodrigues, Amaro F. de Sousa, and Jaime Lloret. 2013. Denial of service mitigation approach for IPv6-enabled smart object networks. Concurrency \& Coput.: Practice \& Experience 25, 1 (Jan. 2013), 129-142.

[17] Edewede Oriwoh and Paul Sant. 2013. The Forensics Edge Management System: A Concept and Design. In IEEE 10th Int. Conf. on Ubiquitous Intelligence and Comput. and IEEE 10th Int. Conf. on Autonomic and Trusted Comput. 544-550.

[18] Yin M. P. Pa, Shogo Suzuki, Katsunari Yoshioka, Tsutomu Matsumoto, Takahiro Kasama, and Christian Rossow. 2016. IoTPOT: A Novel Honeypot for Revealing Current IoT Threats. F. of Inform. Process. 24, 3 (May 2016), 522-533.

[19] George F. Riley and Thomas R. Henderson. 2010. The ns-3 Network Simulator. In Modeling and Tools for Network Simulation, Klaus Wehrle, Mesut GÃijneÅ§, and James Gross (Eds.). Springer Berlin Heidelberg, 15-34.

[20] Na Ruan and Yoshiaki Hori. 2012. DoS attack-tolerant TESLA-based broadcast authentication protocol in Internet of Things. In 2012 International Conference on Selected Topics in Mobile and Wireless Networking. 60-65.

[21] Weisong Shi and Schahram Dustdar. 2016. The Promise of Edge Computing. Computer 49, 5 (May 2016), 78-81.

[22] John Soldatos et al. 2015. OpenIoT: Open Source Internet-of-Things in the Cloud. In Interoperability and Open-Source Solutions for the IoT. Springer, 13-25.

[23] Krushang Sonar and Hardik Upadhyay. 2016. An Approach to Secure Internet of Things Against DDoS. In Proceedings of International Conference on ICT for Sustainable Development. Springer, Singapore, 367-376.

[24] Mališa Vučinić, Bernard Tourancheau, Franck Rousseau, Andrzej Duda, Laurent Damon, and Roberto Guizzetti. 2015. OSCAR: Object security architecture for the Internet of Things. Ad Hoc Networks 32 (Sept. 2015), 3-16.

[25] Kun Wang, Miao Du, Yanfei Sun, Alexey Vinel, and Yan Zhang. 2016. Attack Detection and Distributed Forensics in Machine-to-Machine Networks. IEEE Network 30, 6 (Nov. 2016), 49-55.

[26] Saman Taghavi Zargar, James Joshi, and David Tipper. 2013. A Survey of Defense Mechanisms Against Distributed Denial of Service (DDoS) Flooding Attacks. IEEE Communications Surveys Tutorials 15, 4 (2013), 2046-2069.

[27] Congyingzi Zhang and Robert Green. 2015. Communication Security in Internet of Thing: Preventive Measure and Avoid DDoS Attack over IoT Network. In Proceedings of the 18th Symposium on Communications \& Networking (CNS '15). Society for Computer Simulation International, San Diego, CA, USA, 8-15. 